WSRC-STI-2006-00058

July 20, 2006

WSRC-STI-2006-00058

\title{
Lagrangian Particle Dispersion Model (LPDM) Technical Description
}

Washington Savannah River Company

Savannah River Site

Aiken, SC 29808

Prepared for the U.S. Department of Energy under Contract No. DE-AC09-96SR18500 


\section{Disclaimer}

This report was prepared as an account of work sponsored by an agency of the United States Government. Neither the United States Government nor any agency thereof, nor any of their employees, makes any warranty, express or implied, or assumes any legal liability or responsibility for the accuracy, completeness, or usefulness of any information, apparatus, product, or process disclosed, or represents that its use would not infringe privately owned rights. Reference herein to any specific commercial product, process, or service by trade name, trademark, manufacturer, or otherwise does not necessarily constitute or imply its endorsement, recommendation, or favoring by the United States Government or any agency thereof. The views and opinions of authors expressed herein do not necessarily state or reflect those of the United States Government or any agency thereof. 


\title{
Lagrangian Particle Dispersion Model (LPDM) Technical Description
}

\author{
$07 / 20 / 2006$
}

Kur-Fu Chen, Author, NHSD/NTS

Washington Savannah River Company

B. L. O'Stecn, Technical Review, NHSDNTS

Washington Savannah River Company

Approved by:

R.P. Addis, Manager, NHSDASS

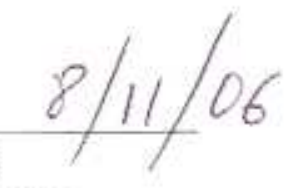

Washington Savannah River Company

Prepared for the U.S. Department of Energy under Contract no. DE-AC09-96SR18500 
WSRC-STI-2006-00058

July 20, 2006

This page intentionally left blank. 
WSRC-STI-2006-00058

July 20, 2006

\section{EXECUTIVE SUMMARY}

The Savannah River National Laboratory (SRNL) uses the Lagrangian Particle Dispersion Model (LPDM) in conjunction with the Regional Atmospheric Modeling System as an operational tool for emergency response consequence assessments for the Savannah River Site (SRS). The LPDM is an advanced stochastic atmospheric transport model used to transport and disperse passive tracers subject to the meteorological field generated by RAMS from sources of varying number and shape. The Atmospheric Technologies Group (ATG) of the SRNL is undertaking the task of reviewing documentation and code for LPDM Quality Assurance (QA). The LPDM QA task will include a model technical description, computer coding descriptions, model applications, and configuration control. This report provides a comprehensive technical description of the LPDM model. 
WSRC-STI-2006-00058

July 20, 2006

\section{TABLE OF CONTENTS}

1. INTRODUCTION 1

2. MDEL EQUATIONS 1

2.1 A System of Equations for $\phi^{\prime} s$

2.2 Solving for $\phi^{\prime} s \quad 6$

2.3 Evaluation of $\gamma^{\prime} s \quad 9$

2.4 Simplified Model 13

3. CONCENTRATION CALCULATION 16

3.1 "Cell" Method 16

3.2 Kernel Method 17

4. SUMMARY 19

5. ACKNOWLEDGMENT 19

$\begin{array}{lr}\text { REFERENCES } & 20\end{array}$ 
WSRC-STI-2006-00058

July 20, 2006

\section{LIST OF FIGURES}

Figure 1: Calculation Sequence for Lagrangian Particle Dispersion Model 


\section{INTRODUCTION}

The Savannah River National Laboratory (SRNL) uses the Lagrangian Particle Dispersion Model (LPDM, Uliasz, 1993) in conjunction with the Regional Atmospheric Modeling System (RAMS, Pielke et al., 1992; Chen, 2005) as an operational tool for emergency response consequence assessments for the Savannah River Site (SRS). The LPDM is an advanced stochastic atmospheric transport model used to transport and disperse passive tracers subject to the meteorological field generated by RAMS from sources of varying number and shape. The Atmospheric Technologies Group (ATG) of the SRNL is undertaking the task of reviewing documentation and code for LPDM Quality Assurance (QA). The LPDM QA task will include a model technical description, computer coding descriptions, model applications, and configuration control. This report provides a comprehensive technical description of the LPDM model.

\section{MODEL EQUATIONS}

The Lagrangian particle model follows a particle in space and time. The instantaneous velocity of the particle is decomposed into a mean and a random (turbulent) component, as shown in Eqs. 1 through 3 in a three dimensional Cartesian coordinate.

$$
\begin{aligned}
& \frac{\partial x}{\partial t}=U=u+u^{\prime \prime}, \\
& \frac{\partial y}{\partial t}=V=v+v^{\prime \prime}, \\
& \frac{\partial z}{\partial t}=W=w+w^{\prime \prime},
\end{aligned}
$$

where $u, v$, and $w$ are mean velocities in the $\mathrm{x}-, \mathrm{y}$ - and $\mathrm{z}$ - direction, respectively. To follow the same notation in the RAMS Technical Description Report (Chen, 2005), the random component is denoted by a double prime. The particle location at $t+\Delta t$ is

$$
\begin{aligned}
& x(t+\Delta t)=x(t)+\left(u+u^{\prime \prime}\right) \Delta t, \\
& y(t+\Delta t)=y(t)+\left(v+v^{\prime \prime}\right) \Delta t, \\
& z(t+\Delta t)=z(t)+\left(w+w^{\prime \prime}\right) \Delta t
\end{aligned}
$$

Markov chain (a special type of stochastic process) deals with a sequence of random events. The occurrence of the current random event is affected by the previous event. The Markov chain for the random velocity of a Lagrangian particle is proposed by Zanetti (1984) as: 
$u^{\prime \prime}(t)=\phi_{1} u^{\prime \prime}(t-\Delta t)+\gamma_{u}$,

$v^{\prime \prime}(t)=\phi_{2} v^{\prime \prime}(t-\Delta t)+\phi_{3} u^{\prime \prime}(t)+\gamma_{v}$,

$w^{\prime \prime}(t)=\phi_{4} w^{\prime \prime}(t-\Delta t)+\phi_{5} v^{\prime \prime}(t)+\phi_{6} u^{\prime \prime}(t)+\gamma_{w}$,

where $\gamma_{u}, \gamma_{v}$ and $\gamma_{w}$ are purely random uncorrelated components, and $\phi_{1}, \ldots, \phi_{6}$ are coefficients which represent the memory effects of the previous time step. The follow section describes the procedures to obtain the coefficients $\left(\phi_{1}, \ldots, \phi_{6}\right)$.

\subsection{A System of Equations for $\phi^{\prime} s$}

This section presents derivations of a system of six equations for the six unknowns $\left(\phi_{1}, \ldots, \phi_{6}\right)$ with the known quantities (variances, co-variances and the Lagrangian integral time scales).

After multiplying Eq. 7 by $u^{\prime \prime}(t-\Delta t)$ and taking the average, one obtains

$\overline{u^{\prime \prime}(t) u^{\prime \prime}(t-\Delta t)}=\overline{\phi_{1} u^{\prime \prime}(t-\Delta t) u^{\prime \prime}(t-\Delta t)}+\overline{\gamma_{u} u^{\prime \prime}(t-\Delta t)}$.

The first term on the right hand of Eq. 10 can be written as:

$\overline{\phi_{1} u^{\prime \prime}(t-\Delta t) u^{\prime \prime}(t-\Delta t)}=\phi_{1} \overline{u^{\prime \prime}(t-\Delta t) u^{\prime \prime}(t-\Delta t)}=\phi_{1} \sigma_{u}^{2}$,

where $\sigma_{u}^{2}$ is the variance of the $\mathrm{u}$ component velocity.

The last term on the right hand of Eq. 10 is

$\overline{\gamma_{u} u^{\prime \prime}(t-\Delta t)}=\gamma_{u} \overline{u^{\prime \prime}(t-\Delta t)}=0$.

Substituting Eqs. 11 and 12 into Eq. 10, we get

$\overline{u^{\prime \prime}(t) u^{\prime \prime}(t-\Delta t)}=\phi_{1} \sigma_{u}^{2}$.

and Eq. 13 can be re-arranged as:

$\phi_{1}=\frac{\overline{u^{\prime \prime}(t) u^{\prime \prime}(t-\Delta t)}}{\sigma_{u}^{2}}=R_{u}$,

where $R_{u}$ is the Lagrangian autocorrelation of wind velocity $u$ for lag time $\Delta t$, and is defined as: 
$R_{u}(\Delta t)=\frac{\overline{u^{\prime \prime}(t) u^{\prime \prime}(t-\Delta t)}}{\sigma_{u}^{2}}=e^{-\frac{\Delta t}{T_{L u}}}$,

where $T_{L u}$ is the Lagrangian integral time scale in the $\mathrm{x}$ direction, and variance $\sigma_{u}^{2}$ is $\overline{u^{\prime \prime} u^{\prime \prime}}$.

The Lagrangian autocorrelation of wind velocity components, $v$, and $w$, are defined in Eqs. 16 and 17 , respectively.

$R_{v}(\Delta t)=\frac{\overline{v^{\prime \prime}(t) v^{\prime \prime}(t-\Delta t)}}{\sigma_{v}^{2}}=e^{-\frac{\Delta t}{T_{L v}}}$,

where $T_{L v}$ is the Lagrangian integral time scale in the y direction, and variance $\sigma_{v}^{2}$ is $\overline{v^{\prime \prime} v^{\prime \prime}}$.

$R_{w}(\Delta t)=\frac{\overline{w^{\prime \prime}(t) w^{\prime \prime}(t-\Delta t)}}{\sigma_{w}^{2}}=e^{-\frac{\Delta t}{T_{L w}}}$

where $T_{L w}$ is the Lagrangian integral time scale in the $\mathrm{z}$ direction, and variance $\sigma_{w}^{2}$ is $\overline{w^{\prime \prime} w^{\prime \prime}}$.

After multiplying Eq. 8 by $v^{\prime \prime}(t-\Delta t)$ and taking the average, we obtain:

$\overline{v^{\prime \prime}(t) v^{\prime \prime}(t-\Delta t)}=\overline{\phi_{2} v^{\prime \prime}(t-\Delta t) v^{\prime \prime}(t-\Delta t)}+\overline{\phi_{3} u^{\prime \prime}(t) v^{\prime \prime}(t-\Delta t)}+\overline{\gamma_{v} v^{\prime \prime}(t-\Delta t)}$.

From Eq. 16, the left hand side of Eq. 18 can be written as:

$\overline{v^{\prime \prime}(t) v^{\prime \prime}(t-\Delta t)}=R_{v} \sigma_{v}^{2}$.

The first term of the right hand side of Eq. 18 is:

$\overline{\phi_{2} v^{\prime \prime}(t-\Delta t) v^{\prime \prime}(t-\Delta t)}=\phi_{2} \sigma_{v}^{2}$.

The second term of the right hand side of Eq. 18 can be derived by multiplying Eq. 7 by $\phi_{3} v^{\prime \prime}(t-\Delta t)$, and then taking the average of the resulting equation, as shown below;

$\overline{u^{\prime \prime}(t) v^{\prime \prime}(t-\Delta t)}=\overline{\phi_{1} u^{\prime \prime}(t-\Delta t) v^{\prime \prime}(t-\Delta t)}+\overline{\gamma_{u} v^{\prime \prime}(t-\Delta t)}=\phi_{1} \overline{u^{\prime \prime} v^{\prime \prime}}$.

From the above relation, it can be shown the second term of the right hand side of Eq. 18 is

$\overline{\phi_{3} u^{\prime \prime}(t) v^{\prime \prime}(t-\Delta t)}=\phi_{3} \phi_{1} \overline{u^{\prime \prime} v^{\prime \prime}}$.

The last term of the right hand side of Eq. 18 is zero. 
After substituting Eqs. 19 through 21 into Eq. 18, it can be written as:

$\phi_{2} \sigma_{v}^{2}+\phi_{1} \phi_{3} \overline{v^{\prime \prime} u^{\prime \prime}}=R_{v} \sigma_{v}^{2}$.

After multiplying Eq. 9 with $w^{\prime \prime}(t-\Delta t)$ and taking the average, we obtain:

$$
\begin{aligned}
& \overline{w^{\prime \prime}(t) w^{\prime \prime}(t-\Delta t)}= \\
& \overline{\phi_{4} w^{\prime \prime}(t-\Delta t) w^{\prime \prime}(t-\Delta t)}+\overline{\phi_{5} v^{\prime \prime}(t) w^{\prime \prime}(t-\Delta t)}+\overline{\phi_{6} u^{\prime \prime}(t) w^{\prime \prime}(t-\Delta t)}+\overline{\gamma_{w} w^{\prime \prime}(t-\Delta t)} .
\end{aligned}
$$

From Eq. 17, the left hand side of Eq. 23 can be written as:

$$
\overline{w^{\prime \prime}(t) w^{\prime \prime}(t-\Delta t)}=R_{w} \sigma_{w}^{2} .
$$

The first term of the right hand side of Eq. 23 is

$$
\overline{\phi_{4} w^{\prime \prime}(t-\Delta t) w^{\prime \prime}(t-\Delta t)}=\phi_{4} \sigma_{w}^{2} \text {. }
$$

By replacing $v^{\prime \prime}$ in the second term of the right hand side of Eq. 23 with Eq. 8 and also utilizing Eq. 7, the second term of the right hand side of Eq. 23 can be simplified as:

$$
\begin{aligned}
& \overline{\phi_{5} v^{\prime \prime}(t) w^{\prime \prime}(t-\Delta t)}=\overline{\phi_{5}\left(\phi_{2} v^{\prime \prime}(t-\Delta t) w^{\prime \prime}(t-\Delta t)+\phi_{3} u^{\prime \prime}(t) w^{\prime \prime}(t-\Delta t)+\gamma_{v} w^{\prime \prime}(t-\Delta t)\right)} \\
& =\overline{\phi_{5} \phi_{2} v^{\prime \prime}(t-\Delta t) w^{\prime \prime}(t-\Delta t)}+\overline{\phi_{5} \phi_{3} u^{\prime \prime}(t) w^{\prime \prime}(t-\Delta t)}+\overline{\phi_{5} \gamma_{v} w^{\prime \prime}(t-\Delta t)} \\
& =\phi_{2} \phi_{5} \overline{v^{\prime \prime} w^{\prime \prime}}+\phi_{5} \phi_{3} \overline{\left(\phi_{1} u^{\prime \prime}(t-\Delta t) w^{\prime \prime}(t-\Delta t)+\gamma_{u} w^{\prime \prime}(t-\Delta t)\right)} \\
& =\phi_{2} \phi_{5} \overline{v^{\prime \prime} w^{\prime \prime}}+\phi_{1} \phi_{3} \phi_{5} \overline{u^{\prime \prime} w^{\prime \prime}}
\end{aligned} .
$$

By replacing $u^{\prime \prime}$ in the third term of the right hand side of Eq. 23 with Eq. 7, the third term of the right hand side of Eq. 23 can be simplified as:

$$
\begin{aligned}
& \overline{\phi_{6} u^{\prime \prime}(t) w^{\prime \prime}(t-\Delta t)}=\overline{\phi_{6}\left(\phi_{1} u^{\prime \prime}(t-\Delta t) w^{\prime \prime}(t-\Delta t)+\gamma_{u} w^{\prime \prime}(t-\Delta t)\right)} . \\
& =\phi_{1} \phi_{6} \overline{u^{\prime \prime} w^{\prime \prime}}
\end{aligned}
$$

The last term of the right hand side of Eq. 23 is zero.

Substituting Eq. 24 through 27 into Eq. 23, we obtain

$$
R_{w} \sigma_{w}^{2}=\phi_{4} \sigma_{w}^{2}+\phi_{5} \phi_{2} \overline{w^{\prime \prime} v^{\prime \prime}}+\phi_{5} \phi_{1} \phi_{3} \overline{u^{\prime \prime} w^{\prime \prime}}+\phi_{6} \phi_{1} \overline{u^{\prime \prime} w^{\prime \prime}} \text {. }
$$


Eq. 28 can be re-arranged as:

$$
R_{w} \sigma_{w}^{2}=\phi_{4} \sigma_{w}^{2}+\phi_{2} \phi_{5} \overline{v^{\prime \prime} w^{\prime \prime}}+\phi_{1}\left(\phi_{3} \phi_{5}+\phi_{6}\right) \overline{u^{\prime \prime} w^{\prime \prime}} \text {. }
$$

It should be noted that the second term of the right hand side of Eq. 29 is different from that inUliasz (1990, Eq. 105), and the author believes that Eq. 105 in Uliasz (1990) is not correct.

Taking the product of Eq, 7 and Eq. 8, we obtain

$$
u^{\prime \prime}(t)\left[\phi_{2} v^{\prime \prime}(t-\Delta t)+\phi_{3} u^{\prime \prime}(t)+\gamma_{v}\right]=\left[\phi_{1} u^{\prime \prime}(t-\Delta t)+\gamma_{u}\right] v^{\prime \prime}(t) \text {. }
$$

Re-arranging and averaging Eq. 30, we get

$$
\begin{aligned}
& \overline{\phi_{2} u^{\prime \prime}(t) v^{\prime \prime}(t-\Delta t)}+\overline{\phi_{3} u^{\prime \prime}(t) u^{\prime \prime}(t)}+\overline{\gamma_{v} u^{\prime \prime}(t)}= \\
& \overline{\phi_{1} u^{\prime \prime}(t-\Delta t) v^{\prime \prime}(t)}+\overline{\gamma_{u} v^{\prime \prime}(t)}
\end{aligned} .
$$

The first term of the left hand side of Eq. 31 can be simplified using Eq. 7 as:

$$
\begin{aligned}
& \overline{\phi_{2} u^{\prime \prime}(t) v^{\prime \prime}(t-\Delta t)}=\phi_{2} \overline{\phi_{1} u^{\prime \prime}(t-\Delta t) v^{\prime \prime}(t-\Delta t)+\gamma_{u} v^{\prime \prime}(t-\Delta t)} \\
& =\phi_{1} \phi_{2} \overline{u^{\prime \prime} v^{\prime \prime}}
\end{aligned}
$$

The second term of the left hand side of Eq. 31 is:

$$
\overline{\phi_{3} u^{\prime \prime}(t) u^{\prime \prime}(t)}=\phi_{3} \sigma_{u}^{2} \text {. }
$$

The first term of the right hand side of Eq. 31 can be simplified using Eq. 7 as:

$$
\overline{u^{\prime \prime}(t) v^{\prime \prime}(t)}=\overline{\phi_{1} u^{\prime \prime}(t-\Delta t) v^{\prime \prime}(t)+\gamma_{u} v^{\prime \prime}(t)} \text {. }
$$

and

$$
\overline{\phi_{1} u^{\prime \prime}(t-\Delta t) v^{\prime \prime}(t)}=\overline{u^{\prime \prime} v^{\prime \prime}}
$$

Substituting Eqs. 32 through 34 into Eq. 31, we obtain

$\phi_{1} \phi_{2} \overline{u^{\prime \prime} v^{\prime \prime}}+\phi_{3} \sigma_{u}^{2}=\overline{u^{\prime \prime} v^{\prime \prime}}$.

Multiplying Eq, 7 and Eq. 9, we obtain

$$
u^{\prime \prime}(t)\left[\phi_{4} w^{\prime \prime}(t-\Delta t)+\phi_{5} v^{\prime \prime}(t)+\phi_{6} u^{\prime \prime}(t)+\gamma_{w}\right]=\left[\phi_{1} u^{\prime \prime}(t-\Delta t)+\gamma_{u}\right] w^{\prime \prime}(t) \text {. }
$$


WSRC-STI-2006-00058

July 20, 2006

Re-arranging and averaging Eq. 36, we get

$\overline{\phi_{4} u^{\prime \prime}(t) w^{\prime \prime}(t-\Delta t)}+\overline{\phi_{5} u^{\prime \prime}(t) v^{\prime \prime}(t)}+\overline{\phi_{6} u^{\prime \prime}(t) u^{\prime \prime}(t)}+\overline{\gamma_{w} u^{\prime \prime}(t)}=\overline{\phi_{1} u^{\prime \prime}(t-\Delta t) w^{\prime \prime}(t)}+\overline{\gamma_{u} w^{\prime \prime}(t)}$.

The first term of the left hand side of Eq. 37 can be simplified by multiplying Eq. 7. We multiply Eq. 7 by $\phi_{4} w^{\prime \prime}(t-\Delta t)$, and then average the resulting equation. The final equation is

$\overline{\phi_{4} u^{\prime \prime}(t) w^{\prime \prime}(t-\Delta t)}=\overline{\phi_{4} \phi_{1} u^{\prime \prime}(t-\Delta t) w^{\prime \prime}(t-\Delta t)+\phi_{4} \gamma_{u} w^{\prime \prime}(t-\Delta t)}$.

The above equation can be simplified as:

$\overline{\phi_{4} u^{\prime \prime}(t) w^{\prime \prime}(t-\Delta t)}=\phi_{4} \phi_{1} \overline{u^{\prime \prime} w^{\prime \prime}}$.

The first term of the right hand side of Eq. 37 can also be simplified from Eq. 7 by multiplying Eq. 7 with $w^{\prime \prime}(t)$, then averaging the resulting equation. The final equation is

$\overline{u^{\prime \prime}(t) w^{\prime \prime}(t)}=\overline{\phi_{1} u^{\prime \prime}(t-\Delta t) w^{\prime \prime}(t)+\gamma_{u} w^{\prime \prime}(t)}$.

The above equation can be re-arranged as

$\overline{\phi_{1} u^{\prime \prime}(t-\Delta t) w^{\prime \prime}(t)}=\overline{u^{\prime \prime} w^{\prime \prime}}$

Substituting Eqs. 38 and 39 into Eq. 37, we obtain

$\phi_{1} \phi_{4} \overline{u^{\prime \prime} w^{\prime \prime}}+\phi_{5} \overline{u^{\prime \prime} v^{\prime \prime}}+\phi_{6} \sigma_{u}^{2}=\overline{u^{\prime \prime} w^{\prime \prime}}$.

Multiplying Eq, 8 and Eq. 9, we get

$$
v^{\prime \prime}(t)\left[\phi_{4} w^{\prime \prime}(t-\Delta t)+\phi_{5} v^{\prime \prime}(t)+\phi_{6} u^{\prime \prime}(t)+\gamma_{w}\right]=\left[\phi_{2} v^{\prime \prime}(t-\Delta t)+\phi_{3} u^{\prime \prime}(t)+\gamma_{v}\right] w^{\prime \prime}(t) .
$$

Re-arranging and averaging Eq. 41, we obtain

$$
\begin{aligned}
& \overline{\phi_{4} v^{\prime \prime}(t) w^{\prime \prime}(t-\Delta t)}+\overline{\phi_{5} v^{\prime \prime}(t) v^{\prime \prime}(t)}+\overline{\phi_{6} u^{\prime \prime}(t) v^{\prime \prime}(t)}+\overline{\gamma_{w} v^{\prime \prime}(t)}=. \\
& \overline{\phi_{2} v^{\prime \prime}(t-\Delta t) w^{\prime \prime}(t)}+\overline{\phi_{3} u^{\prime \prime}(t) w^{\prime \prime}(t)}+\overline{\gamma_{v} w^{\prime \prime}(t)}
\end{aligned}
$$

After replacing the term $v^{\prime \prime}(t)$ in the first term of the left hand side of Eq. 42 with Eq. 8, we obtain the following equation:

$$
\begin{aligned}
& \overline{\phi_{4} v^{\prime \prime}(t) w^{\prime \prime}(t-\Delta t)}=\overline{\phi_{4}\left[\phi_{2} v^{\prime \prime}(t-\Delta t)+\phi_{3} u^{\prime \prime}(t)+\gamma_{v}\right] w^{\prime \prime}(t-\Delta t)} . \\
& =\overline{\phi_{2} \phi_{4} v^{\prime \prime}(t-\Delta t) w^{\prime \prime}(t-\Delta t)}+\overline{\phi_{3} \phi_{4} u^{\prime \prime}(t) w^{\prime \prime}(t-\Delta t)}
\end{aligned}
$$


By substituting Eq. 38 into the last term of the right hand side of the above equation, we get

$$
\overline{\phi_{4} v^{\prime \prime}(t) w^{\prime \prime}(t-\Delta t)}=\phi_{2} \phi_{4} \overline{v^{\prime \prime} w^{\prime \prime}}+\phi_{1} \phi_{3} \phi_{4} \overline{u^{\prime \prime} w^{\prime \prime}}
$$

The first term of the right hand side of Eq. 42 can be obtained from Eq. 8. After multiplying Eq. 8 by $w^{\prime \prime}(t)$, and then averaging the resulting equation, the following equation can be obtained.

$$
\begin{aligned}
& \overline{v^{\prime \prime}(t) w^{\prime \prime}(t)}=\overline{\phi_{2} v^{\prime \prime}(t-\Delta t) w^{\prime \prime}(t)+\phi_{3} u^{\prime \prime}(t) w^{\prime \prime}(t)+\gamma_{v} w^{\prime \prime}(t)} \\
& =\overline{\phi_{2} v^{\prime \prime}(t-\Delta t) w^{\prime \prime}(t)}+\overline{\phi_{3} u^{\prime \prime}(t) w^{\prime \prime}(t)} \\
& =\overline{\phi_{2} v^{\prime \prime}(t-\Delta t) w^{\prime \prime}(t)}+\phi_{3} \overline{u^{\prime \prime} w^{\prime \prime}}
\end{aligned}
$$

The above equation can be re-arranged to obtain the first term of the right hand side of Eq. 42:

$$
\overline{\phi_{2} v^{\prime \prime}(t-\Delta t) w^{\prime \prime}(t)}=\overline{v^{\prime \prime} w^{\prime \prime}}-\phi_{3} \overline{u^{\prime \prime} w^{\prime \prime}} .
$$

Substituting Eqs. 43 and 44 into Eq. 42, we obtain

$$
\phi_{2} \phi_{4} \overline{v^{\prime \prime} w^{\prime \prime}}+\phi_{1} \phi_{3} \phi_{4} \overline{u^{\prime \prime} w^{\prime \prime}}+\phi_{5} \sigma_{v}^{2}+\phi_{6} \overline{u^{\prime \prime} v^{\prime \prime}}=\overline{v^{\prime \prime} w^{\prime \prime}}-\phi_{3} \overline{w^{\prime \prime} u^{\prime \prime}}+\overline{\phi_{3} u^{\prime \prime} w^{\prime \prime}}
$$

which simplified to

$$
\phi_{2} \phi_{4} \overline{v^{\prime \prime} w^{\prime \prime}}+\phi_{1} \phi_{3} \phi_{4} \overline{u^{\prime \prime} w^{\prime \prime}}+\phi_{5} \sigma_{v}^{2}+\phi_{6} \overline{u^{\prime \prime} v^{\prime \prime}}=\overline{v^{\prime \prime} w^{\prime \prime}} \text {. }
$$

\subsection{Solving for $\phi^{\prime} s$}

This section shows the procedures to solve $\phi^{\prime} s$ as a function of variances, co-variances, and the Lagrangian integral time scales. The system of equations to be solved is listed next.

$\phi_{1}=R_{u}$.

$\phi_{2} \sigma_{v}^{2}+\phi_{1} \phi_{3} \overline{v^{\prime \prime} u^{\prime \prime}}=R_{v} \sigma_{v}^{2}$

$R_{w} \sigma_{w}^{2}=\phi_{4} \sigma_{w}^{2}+\phi_{2} \phi_{5} \overline{v^{\prime \prime} w^{\prime \prime}}+\phi_{1}\left(\phi_{3} \phi_{5}+\phi_{6}\right) \overline{u^{\prime \prime} w^{\prime \prime}}$.

$\phi_{1} \phi_{2} \overline{u^{\prime \prime} v^{\prime \prime}}+\phi_{3} \sigma_{u}^{2}=\overline{u^{\prime \prime} v^{\prime \prime}}$

$\phi_{1} \phi_{4} \overline{u^{\prime \prime} w^{\prime \prime}}+\phi_{5} \overline{u^{\prime \prime} v^{\prime \prime}}+\phi_{6} \sigma_{u}^{2}=\overline{u^{\prime \prime} w^{\prime \prime}}$. 
$\phi_{2} \phi_{4} \overline{v^{\prime \prime} w^{\prime \prime}}+\phi_{1} \phi_{3} \phi_{4} \overline{u^{\prime \prime} w^{\prime \prime}}+\phi_{5} \sigma_{v}^{2}+\phi_{6} \overline{u^{\prime \prime} v^{\prime \prime}}=\overline{v^{\prime \prime} w^{\prime \prime}}$.

After substituting Eq. 14 into Eq. 22 and Eq. 35, Eq. 22 becomes

$\phi_{2} \sigma_{v}^{2}+R_{u} \phi_{3} \overline{v^{\prime \prime} u^{\prime \prime}}=R_{v} \sigma_{v}^{2}$,

and Eq. 35 becomes

$$
R_{u} \phi_{2} \overline{u^{\prime \prime} v^{\prime \prime}}+\phi_{3} \sigma_{u}^{2}=\overline{u^{\prime \prime} v^{\prime \prime}} .
$$

Eqs. 47 and 48 can be expressed in a matrix form as:

$$
\left[\begin{array}{cc}
\sigma_{v}^{2} & R_{u} \overline{v^{\prime \prime} u^{\prime \prime}} \\
R_{u} \overline{u^{\prime \prime} v^{\prime \prime}} & \sigma_{u}^{2}
\end{array}\right]\left[\begin{array}{l}
\phi_{2} \\
\phi_{3}
\end{array}\right]=\left[\begin{array}{l}
R_{v} \sigma_{v}^{2} \\
\overline{u^{\prime \prime} v^{\prime \prime}}
\end{array}\right]
$$

The solution of $\phi_{2}$ is

$$
\begin{gathered}
\phi_{2}=\frac{\left|\begin{array}{cc}
\frac{R_{v} \sigma_{v}^{2}}{\overline{u^{\prime \prime} v^{\prime \prime}}} & R_{u} \overline{v^{\prime \prime} u^{\prime \prime}} \\
\sigma_{u}^{2}
\end{array}\right|}{\left|\begin{array}{cc}
\sigma_{v}^{2} & R_{u} \overline{v^{\prime \prime} u^{\prime \prime}} \\
R_{u} \overline{u^{\prime \prime} v^{\prime \prime}} & \sigma_{u}^{2}
\end{array}\right|}=\frac{R_{v} \sigma_{v}^{2} \sigma_{u}^{2}-R_{u} \overline{v^{\prime \prime} u^{\prime \prime}} \overline{u^{\prime \prime} v^{\prime \prime}}}{\sigma_{v}^{2} \sigma_{u}^{2}-R_{u} \overline{v^{\prime \prime} u^{\prime \prime} R_{u}} \overline{u^{\prime \prime} v^{\prime \prime}}}=\frac{R_{v} \sigma_{v}^{2} \sigma_{u}^{2}-R_{u}\left(\overline{u^{\prime \prime} v^{\prime \prime}}\right)^{2}}{\sigma_{v}^{2} \sigma_{u}^{2}-R_{u}^{2}\left(\overline{u^{\prime \prime} v^{\prime \prime}}\right)^{2}} \\
\phi_{2}=\frac{R_{v}-R_{u} \frac{\left(\overline{u^{\prime \prime} v^{\prime \prime}}\right)^{2}}{\sigma_{v}^{2} \sigma_{u}^{2}}}{1-R_{u}^{2} \frac{\left(\overline{u^{\prime \prime} v^{\prime \prime}}\right)^{2}}{\sigma_{v}^{2} \sigma_{u}^{2}}} .
\end{gathered}
$$

The solution of $\phi_{3}$ is

$$
\begin{gathered}
\phi_{3}=\frac{\left|\begin{array}{cc}
R_{u} \overline{u^{\prime \prime} v^{\prime \prime}} & \frac{R_{v} \sigma_{v}^{2}}{u^{\prime \prime} v^{\prime \prime}}
\end{array}\right|}{\left|\begin{array}{cc}
\sigma_{v}^{2} & R_{u} \overline{v^{\prime \prime} u^{\prime \prime}} \\
R_{u} \overline{u^{\prime \prime} v^{\prime \prime}} & \sigma_{u}^{2}
\end{array}\right|}=\frac{\sigma_{v}^{2} \overline{u^{\prime \prime} v^{\prime \prime}}-R_{v} \sigma_{v}^{2} R_{u} \overline{u^{\prime \prime} v^{\prime \prime}}}{\sigma_{v}^{2} \sigma_{u}^{2}-R_{u} \overline{v^{\prime \prime} u^{\prime \prime} R_{u} \overline{u^{\prime \prime} v^{\prime \prime}}}}=\frac{\sigma_{v}^{2} \overline{u^{\prime \prime} v^{\prime \prime}}\left(1-R_{v} R_{u}\right)}{\sigma_{v}^{2} \sigma_{u}^{2}-R_{u}^{2}\left(\overline{u^{\prime \prime} v^{\prime \prime}}\right)^{2}} \\
\phi_{3}=\frac{\left(1-R_{v} R_{u}\right) \frac{\overline{u^{\prime \prime} v^{\prime \prime}}}{\sigma_{u}^{2}}}{1-R_{u}^{2} \frac{\left(\overline{u^{\prime \prime} v^{\prime \prime}}\right)^{2}}{\sigma_{v}^{2} \sigma_{u}^{2}}} .
\end{gathered}
$$


Eq. 29 can be re-arranged as:

$$
\sigma_{w}^{2} \phi_{4}+\left(\phi_{2} \overline{v^{\prime \prime} w^{\prime \prime}}+\phi_{1} \phi_{3} \overline{u^{\prime \prime} w^{\prime \prime}}\right) \phi_{5}+\phi_{1} \overline{u^{\prime \prime} w^{\prime \prime}} \phi_{6}=R_{w} \sigma_{w}^{2} \text {. }
$$

Eq. 46 can be re-arranged as:

$$
\left(\phi_{2} \overline{v^{\prime \prime} w^{\prime \prime}}+\phi_{1} \phi_{3} \overline{u^{\prime \prime} w^{\prime \prime}}\right) \phi_{4}+\sigma_{v}^{2} \phi_{5}+\overline{u^{\prime \prime} v^{\prime \prime}} \phi_{6}=\overline{v^{\prime \prime} w^{\prime \prime}} \text {. }
$$

Eqs. 40, 52 and 53 can be expressed in a matrix form as:

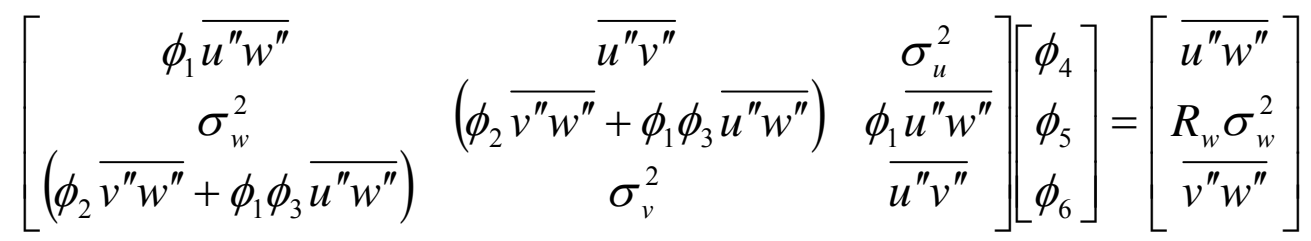

$\phi_{1}, \phi_{2}$ and $\phi_{3}$ in Eq. 54 are given by Eqs. 14, 50 and 51, respectively.

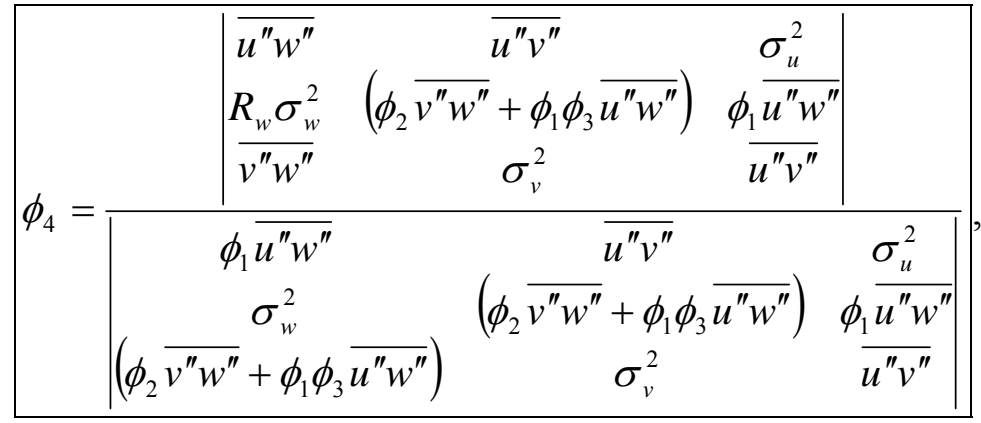

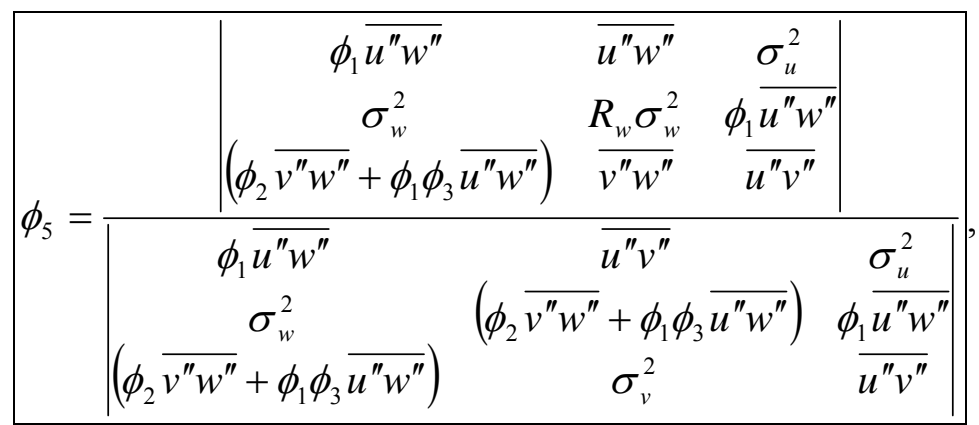




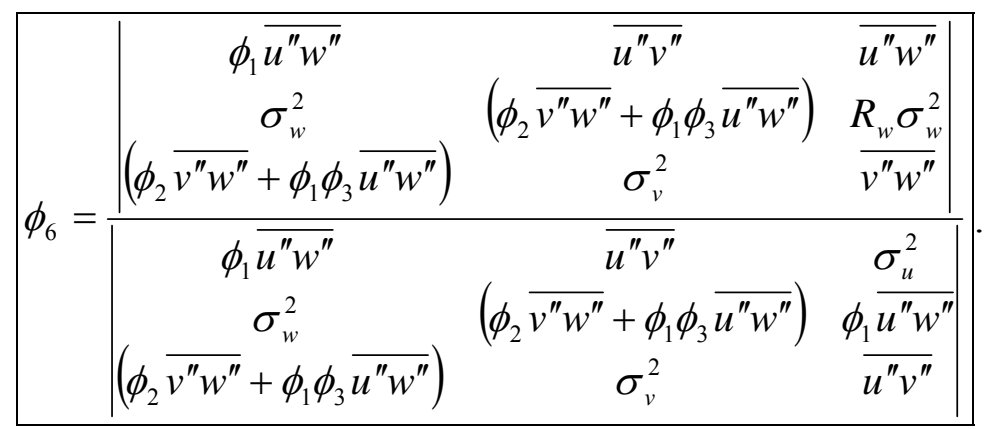

\subsection{Evaluation of the $\gamma$ 's}

The terms, $\gamma_{u}, \gamma_{v}$ and $\gamma_{w}$ are randomly uncorrelated. It is assumed that $\gamma_{u}, \gamma_{v}$ and $\gamma_{w}$ describe a Gaussian distribution, and the mean value of $\gamma_{u}$ and $\gamma_{v}$ is zero. However, the mean value of $\gamma_{w}$ is not zero. This section presents the derivations of the variances of $\gamma_{u}, \gamma_{v}$ and $\gamma_{w}$.

The instantaneous value of $\gamma_{u}$ can be expressed as:

$\gamma_{u}=\overline{\gamma_{u}}+\gamma_{u}^{\prime \prime}=\gamma_{u}^{\prime \prime}$,

where $\overline{\gamma_{u}}$ is the mean value and is zero, and $\gamma_{u}^{\prime \prime}$ is the deviation from the mean.

Substituting Eq. 58 into Eq. 7, one obtains

$\gamma_{u}^{\prime \prime}=u^{\prime \prime}(t)-\phi_{1} u^{\prime \prime}(t-\Delta t)$

Form Eq. 59, the variance of $\gamma_{u}$ is

$$
\begin{aligned}
& \sigma_{\gamma_{u}}^{2}=\overline{\gamma_{u}^{\prime \prime} \gamma_{u}^{\prime \prime}}=\left[u^{\prime \prime}(t)-\phi_{1} u^{\prime \prime}(t-\Delta t)\right]\left[u^{\prime \prime}(t)-\phi_{1} u^{\prime \prime}(t-\Delta t)\right] \\
& =\overline{u^{\prime \prime}(t) u^{\prime \prime}(t)}-2 \phi_{1} \overline{u^{\prime \prime}(t) u^{\prime \prime}(t-\Delta t)}+\phi_{1}^{2} \overline{u^{\prime \prime}(t-\Delta t) u^{\prime \prime}(t-\Delta t)} .
\end{aligned}
$$

From Eq. 7, the term, $\overline{u^{\prime \prime}(t) u^{\prime \prime}(t-\Delta t)}$, can be expressed as:

$$
\overline{u^{\prime \prime}(t) u^{\prime \prime}(t-\Delta t)}=\phi_{1} \overline{u^{\prime \prime}(t-\Delta t) u^{\prime \prime}(t-\Delta t)}+\overline{\gamma_{u} u^{\prime \prime}(t-\Delta t)}=\phi_{1} \sigma_{u}^{2} \text {. }
$$

Substituting Eq. 61 into Eq. 60, the variance of $\gamma_{u}$ is

$$
\begin{aligned}
& \sigma_{\gamma_{u}}^{2}=\sigma_{u}^{2}-2 \phi_{1}^{2} \sigma_{u}^{2}+\phi_{1}^{2} \sigma_{u}^{2}=\sigma_{u}^{2}-\phi_{1}^{2} \sigma_{u}^{2} \\
& \sigma_{\gamma_{u}}^{2}=\sigma_{u}^{2}\left(1-\phi_{1}^{2}\right) .
\end{aligned}
$$


Using the same procedures shown above, the variance of $\gamma_{v}$ can be derived from Eq. 8, as shown below:

$$
\begin{aligned}
& \sigma_{\gamma_{v}}^{2}=\overline{\gamma_{v}^{\prime \prime} \gamma_{v}^{\prime \prime}}=\left[v^{\prime \prime}(t)-\phi_{2} v^{\prime \prime}(t-\Delta t)-\phi_{3} u^{\prime \prime}(t)\right]\left[v^{\prime \prime}(t)-\phi_{2} v^{\prime \prime}(t-\Delta t)-\phi_{3} u^{\prime \prime}(t)\right] \\
& =v^{\prime \prime}(t) v^{\prime \prime}(t)+\phi_{3}^{2} \overline{u^{\prime \prime}(t) u^{\prime \prime}(t)}+\phi_{2}^{2} \overline{v^{\prime \prime}(t-\Delta t) v^{\prime \prime}(t-\Delta t)}-2 \phi_{3} \overline{u^{\prime \prime}(t) v^{\prime \prime}(t)} \\
& +2 \phi_{2} \phi_{3} \overline{u^{\prime \prime}(t) v^{\prime \prime}(t-\Delta t)}-2 \phi_{2} \overline{v^{\prime \prime}(t) v^{\prime \prime}(t-\Delta t)}
\end{aligned}
$$

The above equation can be simplified as:

$$
\sigma_{\gamma_{v}}^{2}=\sigma_{v}^{2}+\phi_{3}^{2} \sigma_{u}^{2}+\phi_{2}^{2} \sigma_{v}^{2}-2 \phi_{3} \overline{u^{\prime \prime} v^{\prime \prime}}+2 \phi_{2} \phi_{3} \overline{u^{\prime \prime}(t) v^{\prime \prime}(t-\Delta t)}-2 \phi_{2} \overline{v^{\prime \prime}(t) v^{\prime \prime}(t-\Delta t)} .
$$

From Eq. 21, the second to last term of the right hand side of Eq. 63 can be expressed as:

$$
2 \phi_{2} \phi_{3} \overline{u^{\prime \prime}(t) v^{\prime \prime}(t-\Delta t)}=2 \phi_{1} \phi_{2} \phi_{3} \overline{u^{\prime \prime} v^{\prime \prime}} .
$$

From Eq. 22, the last term of the right hand side of Eq. 63 can be expressed as:

$$
2 \phi_{2} \overline{v^{\prime \prime}(t) v^{\prime \prime}(t-\Delta t)}=2 \phi_{2}\left[\phi_{2} \sigma_{v}^{2}+\phi_{1} \phi_{3} \overline{v^{\prime \prime} u^{\prime \prime}}\right]=2 \phi_{2}^{2} \sigma_{v}^{2}+2 \phi_{1} \phi_{2} \phi_{3} \overline{v^{\prime \prime} u^{\prime \prime}} .
$$

Substituting Eqs. 64 and 65 into Eq. 63, Eq. 63 becomes:

$$
\begin{aligned}
& \sigma_{\gamma_{v}}^{2}=\sigma_{v}^{2}+\phi_{3}^{2} \sigma_{u}^{2}+\phi_{2}^{2} \sigma_{v}^{2}-2 \phi_{3} \overline{u^{\prime \prime} v^{\prime \prime}}+2 \phi_{1} \phi_{2} \phi_{3} \overline{u^{\prime \prime} v^{\prime \prime}}-2 \phi_{2}^{2} \sigma_{v}^{2}-2 \phi_{1} \phi_{2} \phi_{3} \overline{v^{\prime \prime} u^{\prime \prime}} . \\
& =\sigma_{v}^{2}\left(1-\phi_{2}^{2}\right)+\phi_{3}^{2} \sigma_{u}^{2}-2 \phi_{3} \overline{u^{\prime \prime} v^{\prime \prime}}
\end{aligned}
$$

In order to conform the expression of Eq. 66 with the expression of Eq. 115 in Uliasz 's paper (1990), Eq. 35 is used to modify the last term of the right hand side of Eq. 66. The modified Eq. 66 is

$$
\begin{aligned}
& \sigma_{\gamma_{v}}^{2}=\sigma_{v}^{2}\left(1-\phi_{2}^{2}\right)+\phi_{3}^{2} \sigma_{u}^{2}-2 \phi_{3}\left[\phi_{1} \phi_{2} \overline{u^{\prime \prime} v^{\prime \prime}}+\phi_{3} \sigma_{u}^{2}\right] . \\
& =\sigma_{v}^{2}\left(1-\phi_{2}^{2}\right)+\phi_{3}^{2} \sigma_{u}^{2}-2 \phi_{1} \phi_{2} \phi_{3} \overline{u^{\prime \prime} v^{\prime \prime}}-2 \phi_{3}^{2} \sigma_{u}^{2}
\end{aligned} .
$$

Re-arrange the above equation to obtain the following equation which is the Eq. 115 in Uliasz 's paper (1990):

$$
\sigma_{\gamma_{v}}^{2}=\sigma_{v}^{2}\left(1-\phi_{2}^{2}\right)-\phi_{3}^{2} \sigma_{u}^{2}-2 \phi_{1} \phi_{2} \phi_{3} \overline{u^{\prime \prime} v^{\prime \prime}} \text {. }
$$

The vertical component, $\gamma_{w}$, has a Gaussian distribution with a non-zero mean, and is expressed as:

$$
\gamma_{w}=\overline{\gamma_{w}}+\gamma_{w}^{\prime \prime}
$$


Substituting Eq. 68 into Eq. 9, the term, $\gamma_{w}^{\prime \prime}$ (deviation from the mean), is

$$
\gamma_{w}^{\prime \prime}=w^{\prime \prime}(t)-\phi_{4} w^{\prime \prime}(t-\Delta t)-\phi_{5} v^{\prime \prime}(t)-\phi_{6} u^{\prime \prime}(t)-\overline{\gamma_{w}} .
$$

The variance of $\gamma_{w}$ is

$$
\begin{aligned}
& \sigma_{\gamma_{w}}^{2}=\overline{\gamma_{w}^{\prime \prime} \gamma_{w}^{\prime \prime}} \\
& \left.\left.=w^{\prime \prime}(t)-\phi_{4} w^{\prime \prime}(t-\Delta t)-\phi_{5} v^{\prime \prime}(t)-\phi_{6} u^{\prime \prime}(t)-\overline{\gamma_{w}}\right] w^{\prime \prime}(t)-\phi_{4} w^{\prime \prime}(t-\Delta t)-\phi_{5} v^{\prime \prime}(t)-\phi_{6} u^{\prime \prime}(t)-\overline{\gamma_{w}}\right] \\
& =\overline{w^{\prime \prime}(t) w^{\prime \prime}(t)}+\overline{\phi_{4} w^{\prime \prime}(t-\Delta t) \phi_{4} w^{\prime \prime}(t-\Delta t)}+\overline{\phi_{5} v^{\prime \prime}(t) \phi_{5} v^{\prime \prime}(t)}+\overline{\phi_{6} u^{\prime \prime}(t) \phi_{6} u^{\prime \prime}(t)}+\overline{\overline{\gamma_{w}}} \overline{\gamma_{w}} \\
& -2 \overline{w^{\prime \prime}(t) \phi_{4} w^{\prime \prime}(t-\Delta t)}-2 \overline{w^{\prime \prime}(t) \phi_{5} v^{\prime \prime}(t)}-2 \overline{w^{\prime \prime}(t) \phi_{6} u^{\prime \prime}(t)}-2 \overline{w^{\prime \prime}(t) \overline{\gamma_{w}}} \\
& +2 \overline{\phi_{4} w^{\prime \prime}(t-\Delta t) \phi_{5} v^{\prime \prime}(t)}+2 \overline{\phi_{4} w^{\prime \prime}(t-\Delta t) \phi_{6} u^{\prime \prime}(t)}+2 \overline{\phi_{4} w^{\prime \prime}(t-\Delta t) \overline{\gamma_{w}}} \\
& +2 \overline{\phi_{5} v^{\prime \prime}(t) \phi_{6} u^{\prime \prime}(t)}+2 \overline{\phi_{5} v^{\prime \prime}(t) \overline{\gamma_{w}}}+2 \overline{\phi_{6} u^{\prime \prime}(t) \overline{\gamma_{w}}}
\end{aligned}
$$

Eq. 70 can be simplified as:

$$
\begin{aligned}
& \sigma_{\gamma_{w}}^{2}=\sigma_{w}^{2}+\phi_{4}^{2} \sigma_{w}^{2}+\phi_{5}^{2} \sigma_{v}^{2}+\phi_{6}^{2} \sigma_{u}^{2}+\left(\overline{\gamma_{w}}\right)^{2} \\
& -2 \overline{w^{\prime \prime}(t) \phi_{4} w^{\prime \prime}(t-\Delta t)}-2 \phi_{5} \overline{v^{\prime \prime} w^{\prime \prime}}-2 \phi_{6} \overline{u^{\prime \prime} w^{\prime \prime}} \\
& +2 \phi_{4} \phi_{5} \overline{w^{\prime \prime}(t-\Delta t) v^{\prime \prime}(t)}+2 \phi_{4} \phi_{6} \overline{w^{\prime \prime}(t-\Delta t) u^{\prime \prime}(t)}+2 \phi_{5} \phi_{6} \overline{u^{\prime \prime} v^{\prime \prime}}
\end{aligned} .
$$

The term, $\left(\overline{\gamma_{w}}\right)^{2}$, is a constant which can be lumped into the vertical drift velocity. The term, $2 \overline{w^{\prime \prime}(t) \phi_{4} w^{\prime \prime}(t-\Delta t)}$, can be evaluated by Eq. 29 as:

$$
\begin{aligned}
& 2 \overline{w^{\prime \prime}(t) \phi_{4} w^{\prime \prime}(t-\Delta t)}=2 \phi_{4}\left[\phi_{4} \sigma_{w}^{2}+\phi_{2} \phi_{5} \overline{v^{\prime \prime} w^{\prime \prime}}+\phi_{1}\left(\phi_{3} \phi_{5}+\phi_{6}\right) \overline{u^{\prime \prime} w^{\prime \prime}}\right] \\
& =2 \phi_{4}^{2} \sigma_{w}^{2}+2 \phi_{2} \phi_{4} \phi_{5} \overline{v^{\prime \prime} w^{\prime \prime}}+2 \phi_{1} \phi_{3} \phi_{4} \phi_{5} \overline{u^{\prime \prime} w^{\prime \prime}}+2 \phi_{1} \phi_{4} \phi_{6} \overline{u^{\prime \prime} w^{\prime \prime}}
\end{aligned}
$$

In addition, the term, $2 \phi_{4} \phi_{5} \overline{w^{\prime \prime}(t-\Delta t) v^{\prime \prime}(t)}$, can be evaluated by Eq. 26 as:

$$
\begin{aligned}
& 2 \phi_{4} \phi_{5} \overline{w^{\prime \prime}(t-\Delta t) v^{\prime \prime}(t)}=2 \phi_{4}\left[\phi_{2} \phi_{5} \overline{v^{\prime \prime} w^{\prime \prime}}+\phi_{1} \phi_{3} \phi_{5} \overline{u^{\prime \prime} w^{\prime \prime}}\right] \\
& =2 \phi_{2} \phi_{4} \phi_{5} \overline{v^{\prime \prime} w^{\prime \prime}}+2 \phi_{1} \phi_{3} \phi_{4} \phi_{5} \overline{u^{\prime \prime} w^{\prime \prime}}
\end{aligned}
$$

The term, $2 \phi_{4} \phi_{6} \overline{w^{\prime \prime}(t-\Delta t) u^{\prime \prime}(t)}$, can be evaluated by Eq. 27, as:

$$
2 \phi_{4} \phi_{6} \overline{w^{\prime \prime}(t-\Delta t) u^{\prime \prime}(t)}=2 \phi_{1} \phi_{4} \phi_{6} \overline{u^{\prime \prime} w^{\prime \prime}}
$$


After substituting Eqs. 72 through 74 into Eq. 71, Eq. 71 becomes:

$$
\begin{aligned}
& \sigma_{\gamma_{w}}^{2}=\sigma_{w}^{2}+\phi_{4}^{2} \sigma_{w}^{2}+\phi_{5}^{2} \sigma_{v}^{2}+\phi_{6}^{2} \sigma_{u}^{2}+\left(\overline{\gamma_{w}}\right)^{2} \\
& -\left[2 \phi_{4}^{2} \sigma_{w}^{2}+2 \phi_{2} \phi_{4} \phi_{5} \overline{v^{\prime \prime} w^{\prime \prime}}+2 \phi_{1} \phi_{3} \phi_{4} \phi_{5} \overline{u^{\prime \prime} w^{\prime \prime}}+2 \phi_{1} \phi_{4} \phi_{6} \overline{u^{\prime \prime} w^{\prime \prime}}\right]-2 \phi_{5} \overline{v^{\prime \prime} w^{\prime \prime}}-2 \phi_{6} \overline{u^{\prime \prime} w^{\prime \prime}} . \\
& +\left[2 \phi_{2} \phi_{4} \phi_{5} \overline{v^{\prime \prime} w^{\prime \prime}}+2 \phi_{1} \phi_{3} \phi_{4} \phi_{5} \overline{u^{\prime \prime} w^{\prime \prime}}\right]+2 \phi_{1} \phi_{4} \phi_{6} \overline{u^{\prime \prime} w^{\prime \prime}}+2 \phi_{5} \phi_{6} \overline{u^{\prime \prime} v^{\prime \prime}}
\end{aligned}
$$

After re-grouping, we obtain

$$
\begin{aligned}
& \sigma_{\gamma_{w}}^{2}=\sigma_{w}^{2}+\phi_{5}^{2} \sigma_{v}^{2}+\phi_{6}^{2} \sigma_{u}^{2} \\
& -\phi_{4}^{2} \sigma_{w}^{2}-2 \phi_{1} \phi_{3} \phi_{4} \phi_{5} \overline{u^{\prime \prime} w^{\prime \prime}}-2 \phi_{1} \phi_{4} \phi_{6} \overline{u^{\prime \prime} w^{\prime \prime}}-2 \phi_{5} \overline{v^{\prime \prime} w^{\prime \prime}}-2 \phi_{6} \overline{u^{\prime \prime} w^{\prime \prime}} . \\
& +2 \phi_{1} \phi_{3} \phi_{4} \phi_{5} \overline{u^{\prime \prime} w^{\prime \prime}}+2 \phi_{1} \phi_{4} \phi_{6} \overline{u^{\prime \prime} w^{\prime \prime}}+2 \phi_{5} \phi_{6} \overline{u^{\prime \prime} v^{\prime \prime}}
\end{aligned}
$$

The term, $2 \phi_{6} \overline{u^{\prime \prime} w^{\prime \prime}}$, in Eq. 76 can be replaced by Eq. 40 and we get:

$$
2 \phi_{6} \overline{u^{\prime \prime} w^{\prime \prime}}=2 \phi_{6}\left[\phi_{1} \phi_{4} \overline{u^{\prime \prime} w^{\prime \prime}}+\phi_{5} \overline{u^{\prime \prime} v^{\prime \prime}}+\phi_{6} \sigma_{u}^{2}\right]=2 \phi_{1} \phi_{4} \phi_{6} \overline{u^{\prime \prime} w^{\prime \prime}}+2 \phi_{5} \phi_{6} \overline{u^{\prime \prime} v^{\prime \prime}}+2 \phi_{6}^{2} \sigma_{u}^{2} \text {. }
$$

Also the term, $2 \phi_{5} \overline{v^{\prime \prime} w^{\prime \prime}}$, after being replaced by Eq. 46 , becomes:

$$
\begin{aligned}
& 2 \phi_{5} \overline{v^{\prime \prime} w^{\prime \prime}}=2 \phi_{5}\left[\phi_{2} \phi_{4} \overline{v^{\prime \prime} w^{\prime \prime}}+\phi_{1} \phi_{3} \phi_{4} \overline{u^{\prime \prime} w^{\prime \prime}}+\phi_{5} \sigma_{v}^{2}+\phi_{6} \overline{u^{\prime \prime} v^{\prime \prime}}\right] \\
& =2 \phi_{2} \phi_{4} \phi_{5} \overline{v^{\prime \prime} w^{\prime \prime}}+2 \phi_{1} \phi_{3} \phi_{4} \phi_{5} \overline{u^{\prime \prime} w^{\prime \prime}}+2 \phi_{5}^{2} \sigma_{v}^{2}+2 \phi_{6} \phi_{5} \overline{u^{\prime \prime} v^{\prime \prime}}
\end{aligned}
$$

After substituting Eqs. 77 and 78 into Eq. 76, Eq. 76 becomes:

$$
\begin{aligned}
& \sigma_{\gamma_{w}}^{2}=\sigma_{w}^{2}+\phi_{5}^{2} \sigma_{v}^{2}+\phi_{6}^{2} \sigma_{u}^{2} \\
& -\phi_{4}^{2} \sigma_{w}^{2}-2 \phi_{1} \phi_{3} \phi_{4} \phi_{5} \overline{u^{\prime \prime} w^{\prime \prime}}-2 \phi_{1} \phi_{4} \phi_{6} \overline{u^{\prime \prime} w^{\prime \prime}} \\
& -2 \phi_{2} \phi_{4} \phi_{5} \overline{v^{\prime \prime} w^{\prime \prime}}-2 \phi_{1} \phi_{3} \phi_{4} \phi_{5} \overline{u^{\prime \prime} w^{\prime \prime}}-2 \phi_{5}^{2} \sigma_{v}^{2}-2 \phi_{6} \phi_{5} \overline{u^{\prime \prime} v^{\prime \prime}} . \\
& -2 \phi_{1} \phi_{4} \phi_{6} \overline{u^{\prime \prime} w^{\prime \prime}}-2 \phi_{5} \phi_{6} \overline{u^{\prime \prime} v^{\prime \prime}}-2 \phi_{6}^{2} \sigma_{u}^{2} \\
& +2 \phi_{1} \phi_{3} \phi_{4} \phi_{5} \overline{u^{\prime \prime} w^{\prime \prime}}+2 \phi_{1} \phi_{4} \phi_{6} \overline{u^{\prime \prime} w^{\prime \prime}}+2 \phi_{5} \phi_{6} \overline{u^{\prime \prime} v^{\prime \prime}}
\end{aligned}
$$

Eq. 79 can be simplified as:

$$
\sigma_{\gamma_{w}}^{2}=\sigma_{w}^{2}\left(1-\phi_{4}^{2}\right)-\phi_{5}^{2} \sigma_{v}^{2}-\phi_{6}^{2} \sigma_{u}^{2}-2 \phi_{5} \phi_{6} \overline{u^{\prime \prime} v^{\prime \prime}}-2 \phi_{1} \phi_{4}\left[\phi_{3} \phi_{5}+\phi_{6}\right] \overline{u^{\prime \prime} w^{\prime \prime}}-2 \phi_{2} \phi_{4} \phi_{5} \overline{v^{\prime \prime} w^{\prime \prime}} \text {. }
$$

It should be noted that the second term of the right hand side of Eq. 80 is different from that in the Eq. 116 of Uliasz (1990), and the author believes that Eq. 116 in Uliasz (1990) is not correct. 
WSRC-STI-2006-00058

July 20, 2006

The random fluctuations are generated at each time step as:

$\gamma_{u}=\sigma_{\gamma_{u}} \eta_{u}$

$\gamma_{v}=\sigma_{\gamma_{v}} \eta_{v}$

and

$\gamma_{w}=\sigma_{\gamma_{w}} \eta_{w}+\omega_{d}$

where $\eta_{u}, \eta_{v}, \eta_{w}$ are random numbers from a normalized Gaussian distribution, and $\omega_{d}$ is a vertical drift velocity.

The Lagrangian time scales are derived from the diagonal elements of the eddy diffusivity tensor, $K_{x x}, K_{y y}$, and $K_{z z}$ as:

$T_{L u}=\frac{K_{x x}}{\sigma_{u}^{2}}$

$T_{L v}=\frac{K_{y y}}{\sigma_{v}^{2}}$

$T_{L w}=\frac{K_{z z}}{\sigma_{w}^{2}}$

The eddy diffusivity tensor is derived from the second order closure scheme in the RAMS model.

The time step, $\Delta t$, used in the LPDM calculation varies in inhomogeneous turbulent flow and depends on $T_{L w}$ :

$$
\Delta t=\max \left(0.1 T_{L w}, \Delta t_{\min }\right) .
$$

The minimum time step, $\Delta t_{\text {min }}$, is an arbitrarily pre-scribed input value to avoid zero time step near the ground surface.

\subsection{Simplified Model}

If the covariance terms of the turbulence are ignored, the model is simplified. From Eq. 50, $\phi_{2}$ is 


$$
\phi_{2}=\frac{R_{v}-R_{u} \frac{\left(\overline{u^{\prime \prime} v^{\prime \prime}}\right)^{2}}{\sigma_{v}^{2} \sigma_{u}^{2}}}{1-R_{u}^{2} \frac{\left(\overline{u^{\prime \prime} v^{\prime \prime}}\right)^{2}}{\sigma_{v}^{2} \sigma_{u}^{2}}}=R_{v} .
$$

From Eq. 51, $\phi_{3}$ is

$$
\phi_{3}=\frac{\left(1-R_{v} R_{u}\right) \frac{\overline{u^{\prime \prime} v^{\prime \prime}}}{\sigma_{u}^{2}}}{1-R_{u}^{2} \frac{\left(\overline{u^{\prime \prime} v^{\prime \prime}}\right)^{2}}{\sigma_{v}^{2} \sigma_{u}^{2}}}=0 .
$$

From Eq. 55, $\phi_{4}$ is

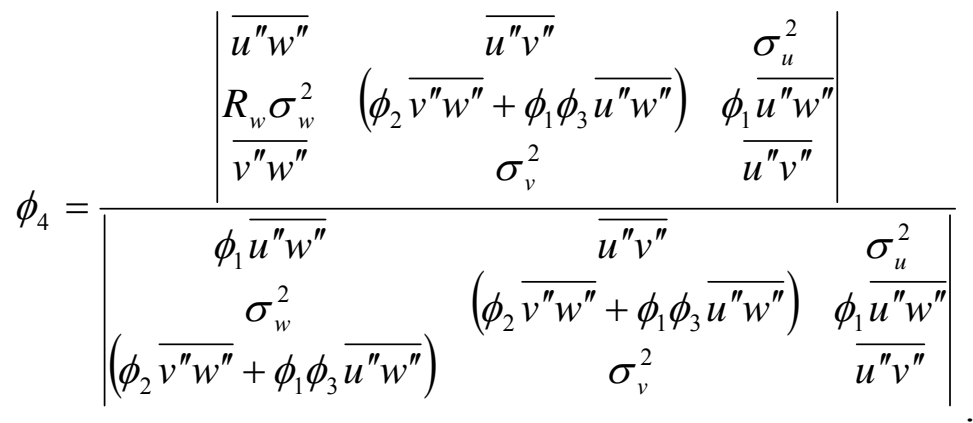

$$
\begin{aligned}
& =\frac{\left|\begin{array}{ccc}
0 & 0 & \sigma_{u}^{2} \\
R_{w} \sigma_{w}^{2} & 0 & 0 \\
0 & \sigma_{v}^{2} & 0
\end{array}\right|}{\left|\begin{array}{ccc}
0 & 0 & \sigma_{u}^{2} \\
\sigma_{w}^{2} & 0 & 0 \\
0 & \sigma_{v}^{2} & 0
\end{array}\right|}=\frac{R_{w} \sigma_{w}^{2} \sigma_{v}^{2} \sigma_{u}^{2}}{\sigma_{w}^{2} \sigma_{v}^{2} \sigma_{u}^{2}}=R_{w}
\end{aligned}
$$

From Eq. $56, \phi_{5}$ is 


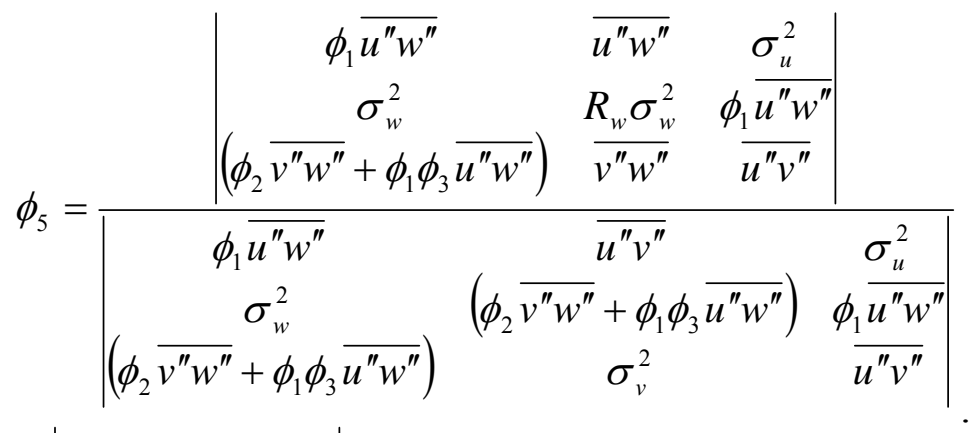

$$
\begin{aligned}
& =\frac{\left|\begin{array}{ccc}
0 & 0 & \sigma_{u}^{2} \\
\sigma_{w}^{2} & R_{w} \sigma_{w}^{2} & 0 \\
0 & 0 & 0
\end{array}\right|}{\left|\begin{array}{ccc}
0 & 0 & \sigma_{u}^{2} \\
\sigma_{w}^{2} & 0 & 0 \\
0 & \sigma_{v}^{2} & 0
\end{array}\right|}=0
\end{aligned}
$$

From Eq. 57, $\phi_{6}$ is

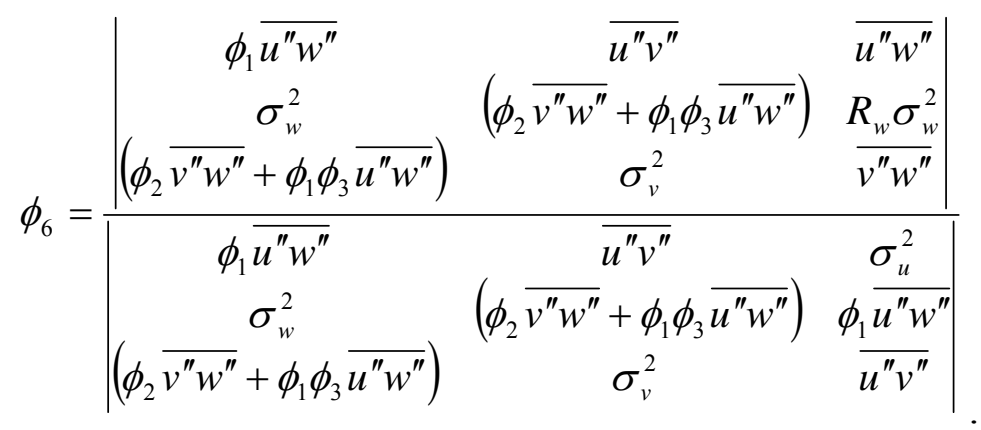

$$
=\frac{\left|\begin{array}{ccc}
0 & 0 & 0 \\
\sigma_{w}^{2} & 0 & R_{w} \sigma_{w}^{2} \\
0 & \sigma_{v}^{2} & 0
\end{array}\right|}{\left|\begin{array}{ccc}
0 & 0 & \sigma_{u}^{2} \\
\sigma_{w}^{2} & 0 & 0 \\
0 & \sigma_{v}^{2} & 0
\end{array}\right|}=0
$$

Substituting Eqs. 88 and 89 into Eq. 67, Eq. 67 becomes:

$\sigma_{\gamma_{v}}^{2}=\sigma_{v}^{2}\left(1-R_{v}^{2}\right)$,

and Eq. 82 becomes;

$$
\gamma_{v}=\sigma_{\gamma_{u}} \eta_{u}=\sigma_{v}\left(1-R_{v}^{2}\right)^{\frac{1}{2}} \eta_{v}
$$


Substituting Eqs. 90, through 92 into Eq. 80, Eq. 80 becomes:

$$
\sigma_{\gamma_{w}}^{2}=\sigma_{w}^{2}\left(1-R_{w}^{2}\right)
$$

and Eq. 83 becomes:

$$
\gamma_{w}=\sigma_{\gamma_{w}} \eta_{w}+\omega_{d}=\sigma_{w}\left(1-R_{w}^{2}\right)^{\frac{1}{2}} \eta_{w}+\omega_{d} .
$$

Substituting Eqs. 14 and 62 into Eq. 81, Eq. 81 becomes:

$$
\gamma_{u}=\sigma_{\gamma_{u}} \eta_{u}=\sigma_{u}\left(1-R_{u}^{2}\right)^{\frac{1}{2}} \eta_{u}
$$

Substituting Eqs. 14 and 95 into Eq. 7, Eq. 7 becomes:

$$
u^{\prime \prime}(t)=R_{u} u^{\prime \prime}(t-\Delta t)+\sigma_{u}\left(1-R_{u}^{2}\right)^{\frac{1}{2}} \eta_{u}
$$

Substituting Eqs. 88, 89 and 93 into Eq. 8, Eq. 8 becomes:

$$
v^{\prime \prime}(t)=R_{v} v^{\prime \prime}(t-\Delta t)+\sigma_{v}\left(1-R_{v}^{2}\right)^{\frac{1}{2}} \eta_{v} .
$$

Substituting Eqs. 90, 91, 92 and 94 into Eq. 9, Eq. 9 becomes:

$$
w^{\prime \prime}(t)=R_{w} w^{\prime \prime}(t-\Delta t)+\sigma_{w}\left(1-R_{w}^{2}\right)^{\frac{1}{2}} \eta_{w}+\omega_{d} .
$$

Eqs. 96, 97 and 98 which neglect the covariance terms are used in the current LPDM model. The LPDM is going to be upgraded to include all the covariance terms.

\section{CONCENTRATION CALCULATION}

\section{1 “Cell” Method}

In the Lagrangian particle transport calculation, the calculated particle concentration at a given time and space can be determined by counting the number of particles in a sampling volume, described as:

$C(x, y, z, t)=\frac{\sum_{i=1}^{N} m_{i}}{(\Delta x \Delta y \Delta z)_{l}}$, 
where $(\Delta x \Delta y \Delta z)_{l}$ is a sampling volume at location $l, m_{i}$ is the mass of particle $i$, and $\mathrm{N}$ is the number of particles in the sampling volume. Normally known as the "Cell" method, Eq. 99 shows that the calculated particle concentration is dependent on the number of particles to be simulated and the sampling volume size. If the sampling volume is too large, the calculated concentration can be over-smoothed. On the other hand, if the sampling volume is too small, the calculated concentration is very noisy. One may increase the number of sampling particles to minimize the noise. However, this increase in sampling particles results in higher computational cost..

\subsection{Kernel Method}

To improve the computational efficiency, Yamada and Bunker (1988) proposed a kernel density estimator method to calculate the particle concentration. The main idea of the kernel density estimate method is that statistically, one could imaging that there would be a probability distribution of particles associated with a sampling particle at location $i$. Yamada and Bunker (1988) assumed this probability distribution is a Gaussian distribution and the mean location of this distribution is at the sampling particle location $i$. This distribution would contribute some particle concentrations at a location $l$. Therefore, the particle concentration at the location $l$ could be estimated by summing the contributions from all the sampling particles in the space. This kernel method does not need a sampling volume and can produce a smooth concentration distribution with a much smaller number of sampling particles. Therefore, it would improve the computational efficiency significantly. The mathematical expression of this kernel method is

$$
C\left(l_{x}, l_{y}, l_{z}\right)=m \sum_{i=1}^{N} \frac{1}{h_{x i} h_{y i} h_{z i}}\left[K\left(\frac{l_{x}-X_{i}}{h_{x i}}, \frac{l_{y}-Y_{i}}{h_{y i}}, \frac{l_{z}-Z_{i}}{h_{z i}}\right)+K\left(\frac{l_{x}-X_{i}}{h_{x i}}, \frac{l_{y}-Y_{i}}{h_{y i}}, \frac{l_{z}+Z_{i}}{h_{z i}}\right)\right],
$$

where

$C\left(l_{x}, l_{y}, l_{z}\right)=$ particle concentration at location $l$,

$\left(X_{i}, Y_{i}, Z_{i}\right)=$ coordinates of the $i$-th sampling particle,

$m=$ particle mass,

$N=$ number of the sampling particle,

$h_{x i}, h_{y i}, h_{z i}=$ parameters to determine the distribution bandwidth in $\mathrm{X}, \mathrm{Y}, \mathrm{Z}$ direction

respectively,

$K=$ probability distribution function.

The last term in Eq. 100 accounts for the particles reflected from the ground surface.

Yamada and Bunker (1988), and Uliasz (1990) assumed that the bandwidth $\left(h_{x i}, h_{y i}, h_{z i}\right)$ are calculated separately for each particle, and were assumed to be proportional to the standard deviation $\left(\sigma_{x i}, \sigma_{y i}, \sigma_{z i}\right)$ of a Gaussian puff with a center at the particle coordinates:

$h_{x i}=a \sigma_{x i}$ 
$h_{y i}=a \sigma_{y i}$,

$h_{z i}=a \sigma_{z i}$,

where a is a proportional constant. Based on Taylor's homogeneous diffusion theory, Yamada and Bunker (1988) derived the standard deviations $\left(\sigma_{x i}, \sigma_{y i}, \sigma_{z i}\right)$ by time integration of the velocity variances encountered during the history of the particle. For example, $\sigma_{y i}$ for the $i$-th particle (the subscript $i$ is dropped in the following derivations for convenience) is

$$
\sigma_{y}^{2}=2 \sigma_{v}^{2} \int_{0}^{t} \int_{0}^{\tau} R_{v}(\zeta) d \zeta d \tau
$$

Substituting Eq. 16 into Eq. 104, we obtain:

$$
\begin{aligned}
& \sigma_{y}^{2}=2 \sigma_{v}^{2} \int_{0}^{t} \int_{0}^{\tau} e^{-\frac{\zeta}{T_{L v}}} d \zeta d \tau=\left.2 \sigma_{v}^{2} \int_{0}^{t}\left(-T_{L v} e^{-\frac{\zeta}{T_{L v}}}\right)\right|_{0} ^{\tau} d \tau \\
& =2 \sigma_{v}^{2} \int_{0}^{t}\left(T_{L V}-T_{L V} e^{-\frac{\tau}{T_{L V}}}\right) d \tau=2 \sigma_{v}^{2}\left(T_{L V} t+T_{L v}^{2} e^{-\frac{t}{T_{L V}}}-T_{L v}^{2}\right) \\
& =2 \sigma_{v}^{2} T_{L V}\left(t+T_{L V} e^{-\frac{t}{T_{L V}}}-T_{L V}\right)
\end{aligned}
$$

For $t \leq 2 T_{L V}$, the term $T_{L V} e^{-\frac{t}{T_{L V}}}$ can be approximated as $T_{L V}$. Therefore, Eq. 105 is approximated as:

$$
\sigma_{y}^{2}=2 \sigma_{v}^{2} T_{L V}\left(t+T_{L V} e^{-\frac{t}{T_{L V}}}-T_{L V}\right) \cong 2 \sigma_{v}^{2} T_{L V} t \approx \sigma_{v}^{2} t^{2}
$$

From Eq. 106, the standard deviation can be approximated as:

$$
\sigma_{y}=\sigma_{v} t
$$

For $t>2 T_{L v}$, the term $T_{L V} e^{-\frac{t}{T_{L V}}}$ can be approximated as zero. Therefore, Eq. 105 is approximated as:

$$
\sigma_{y}^{2}=2 \sigma_{v}^{2} T_{L V}\left(t+T_{L V} e^{-\frac{t}{T_{L V}}}-T_{L V}\right) \cong 2 \sigma_{v}^{2} T_{L V}\left(t-T_{L V}\right) \approx 2 \sigma_{v}^{2} T_{L V} t
$$


From Eq. 108, the standard deviation can be approximated as:

$\sigma_{y}^{2}=2 \sigma_{v}^{2} T_{L V} t$

The standard deviation in the y-direction is summarized as:

$$
\begin{array}{ll}
\sigma_{y}=\sigma_{v} t & \text { for } t \leq 2 T_{L v} \\
\sigma_{y}^{2}=2 \sigma_{v}^{2} T_{L V} t & \text { for } t>2 T_{L v}
\end{array}
$$

It is assumed that this theory ia applicable over a short time period, such as the time step $\Delta \mathrm{t}$ used in the particle transport simulations:

$$
\begin{array}{ll}
\sigma_{y}(t+\Delta t)=\sigma_{y}(t)+\sigma_{v} \Delta t & \text { for } t \leq 2 T_{L v} \\
\sigma_{y}^{2}(t+\Delta t)=\sigma_{y}^{2}(t)+2 \sigma_{v}^{2} T_{L V} \Delta t & \text { for } t>2 T_{L v}
\end{array}
$$

The standard deviations for the other directions $\left(\sigma_{x i}\right.$, and $\sigma_{z i}$ ) can be derived in a similar manner.

The Gaussian distribution function was used for the distribution function $K$ by Yamada and Bunker (1988), and Uliasz (1990). After substituting the distribution function, $K$, in Eq. 100 with the Gaussian distribution function, the concentration equation, Eq. 100 becomes:

$$
C\left(l_{x}, l_{y}, l_{z}\right)=m \sum_{i=1}^{N} \frac{\exp \left(-\frac{1}{2} r_{x}^{2}\right) \exp \left(-\frac{1}{2} r_{y}^{2}\right)}{(2 \pi)^{\frac{3}{2}} h_{x i} h_{y i} h_{z i}}\left[\exp \left(-\frac{1}{2}\left(\frac{l_{z}-Z_{i}}{h_{z i}}\right)^{2}\right)+\exp \left(-\frac{1}{2}\left(\frac{l_{z}+Z_{i}}{h_{z i}}\right)^{2}\right)\right]
$$

where

$r_{x}=\frac{l_{x}-X_{i}}{h_{x i}}$, and $r_{y}=\frac{l_{y}-Y_{i}}{h_{y i}}$

Problems have been encountered when trying to implement the kernel method estimator. Therefore, NTS generally uses the "Cell" method (Eq. 99).

\section{SUMMARY}

The tedious derivations are presented in Sections 2 to 4 . To provide a clear picture of the Lagrangian particle dispersion model, Table 1 summarizes the calculation sequence of the LPDM. The block at the beginning or the end of an arrow contain the equations used to 
calculate the required parameters. The block next to an arrow contain the output parameters from the block at the beginning of that arrow, and those output parameters become input to the block at the end of that arrow.

\section{ACKNOWLEDGMENT}

The author of this report would like to extend his appreciation to Lance O'Steen and Robert Buckley, for the invaluable discussions and numerous consultations. 


\section{REFERENCES}

Chen, Kuo-Fu (2005): Regional Atmospheric Modeling System (RAMS) Technical Description (U). WSRC-TR-2005-00499, Savannah River National Laboratory, Savannah River Site, Aiken, $\mathrm{SC}$.

Pielke, R.A., W.R. Cotton, R.L. Walko, C.J. Tremback, W.A. Lyons, L.D. Grasso, M.E. Nicholls, M.D. Moran, D.A. Wesley, T.J. Lee, and J.H. Copeland (1992): A comprehensive meteorological modeling system-RAMS. Meteor. Atmos. Phys., 49, 69-91.

Uliasz, M., (1990): The Mesoscale Dispersion Modeling System. Department of Atmospheric Science, Colorado State University, Fort Collins, CO 80523.

Uliasz, M. (1993): The atmospheric mesoscale dispersion modeling system. J. Appl. Meteor., 32, 139-149.

Yamada, T., S. Bunker, (1988): Development of a nested grid, second moment turbulence closure model and application to the ASCOT Brush Creek data simulation. J. Appl. Meteor., 27, 562-578.

Zanetti, P., (1984): New Monte Carlo scheme for simulating Lagrangian particle diffusion with wind shear effects. Appl. Math. Modeling, 8, 188-192. 
Figure 1 Calculation Sequence for Lagrangian Particel Dispersion Model

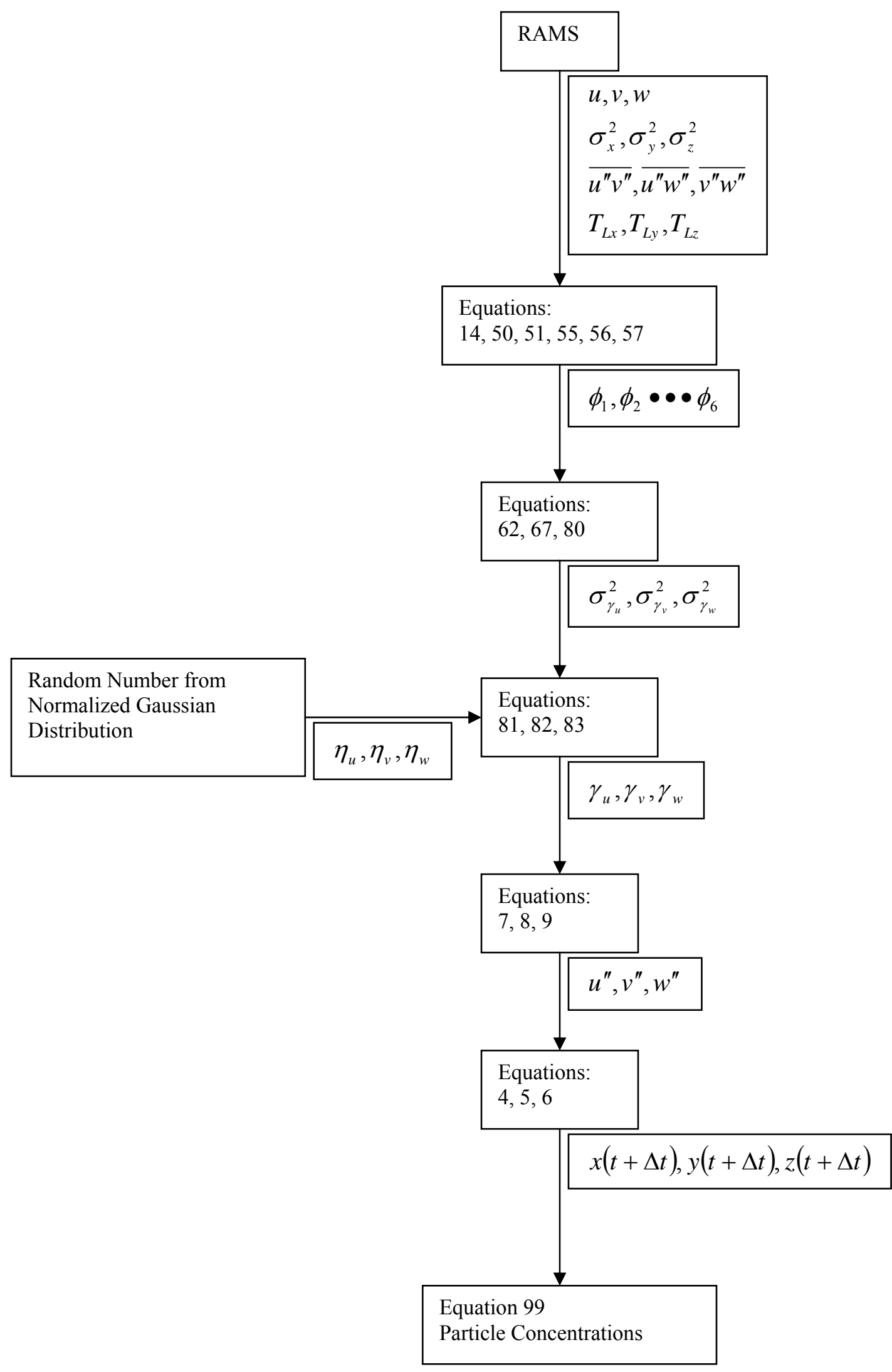




\section{DISTRIBUTION}

\section{SAVANNAH RIVER SITE}
A. L. Boni, 735-A
R. P. Addis, 773-A
R. L. Buckley, 773-A
Kuo-Fu Chen, 773-A
D. P. Griggs, 735-A
C. H. Hunter, 773-A
R. J. Kurzeja, 773-A
B. L. O'Steen, 773-A
M. J. Parker, 773-A
NTS Records (5), 773-A 